\title{
BIOMIMICRY PERMEATED ARCHITECTURE PEDAGOGY A METHOD OF INVESTIGATING BIO-MIMICRY AND DIGITAL TECHNIQUES IN TEH ARCHITECTUREAL DESGIN STUDIOS
}

\author{
Quratulain Asghar* \\ Syed M uhammad Zille Ali Naqvi**
}

\begin{abstract}
In the emerging era of technology, the architectural world is taking inspiration from nature for solutions to its problems, which involve the study of natural design systems and various processes. This research investigation, carried out in the fourth year architecture design studio, aims to investigate bio-mimicry as a development process, involving it for architectural design. It also attempts to study innovation by integrating the digital tools like Rhino, Grasshopper, Ladybug and Para Cloud Gem. A new understanding of solving design issues with the help of natural processes and phenomena is the basic aim.
\end{abstract}

Natural systems offer design strategies to improve design thinking due to the availability of extensive repertoire; which makes incorporating multi-functional and self-organized biomimetic principles into the design process a requirement. This discusses an undergraduate design studio titled "Digital Design through Bio-mimicry" which was taught by the author in an architectural degree program at the University of Engineering and Technology Lahore, Department of Architecture in Spring 2018. Following the exploration of individual research topics, the findings were implemented into design solutions. It has been a critical challenge for the author to increase the skill of students about biomimetic thinking, making them learn about how to handle digital tool's performance issues, as well as making them work on the development of interesting form generation. The challenges encountered in the teaching process and future lines of the work are discussed in this paper.

Keywords: Biomimetic processes, Architectural education, Digital Techniques, Vertical Landscape, Computer-Aided Architectural Design.

\section{INTRODUCTION}

The bio-mimicry approach to education includes conducting short-term research on any selected phenomena in nature by the students themselves. The goal of the biomimicrybased studio was to develop innovative structures strongly inspired by a certain phenomenon in nature. The students' work helped to devise a framework for the studio teaching with bio-mimicry and digital tools.

The main aim of the studio was to conduct research based on the relationship between a natural phenomenon, processes, and CAAD (Computer Aided Architectural Design). The knowledge gained through studying a natural phenomenon or process was then used to interpret architectural solutions.

According to Oxman, 2007, processes are becoming far more interesting than ideas. Processes of generation are synthesized as a kind of accelerated motion, adding information integrally to the construction. This sequential, integrative addition produces more ambiguous effects, more capable of resonating on different levels than straightforward ideological statements, metaphors, allegories or reproductions.

Students were given well-defined and focused studio instructions that served as a reference. Through this exercise, a new working methodology was introduced in academia which was a fusion of traditional research practices and explorations in technology in architecture. The study area was demarcated and based on three basic components; namely, study of natural processes, study of working principles natural processes and study of natural phenomena. Nature is the cradle for some very interesting processes and phenomena. The attempt here was to learn from these and draw useful inferences in architecture. The objective of the project was to study the working principles underlying digital

\footnotetext{
* Quraulain Asghar, Assistant Professor Department of Architecture, University of Engineering and Technology Lahore.

Email Correspondance: qurtulainasghar@gmail.com

** Syed Muhammad Zille Ali Naqvi: Principal Architect at Studio Arch, Architectural Consultancy Firm in Lahore.
} 
systems, such as information handling, visual programming, and modeling techniques. Each student explored these disciplines in their own way to develop an architectural form.

In the architectural curriculum, currently architectural design and learning how to handle digital tools are not taught separately. The strong categorical interactions between the two have become obligatory. In this exercise, the students were mostly considered to bring logic in the two fields by themselves. This exercise also intended to explore the computer models of various types and how the manner in which basic issues in design development can change with time, for example, space generation, assessment and eventually the role of the architect in the building design process.

The combination of all three components helped the instructor to explore various aspects of using digital tools in the studio. The approach and results of three best explorations are presented in this paper.

\section{Literature Review}

The search for new educational frameworks is due to the pedagogically unique impacts of digital design. Various researchers and educators have begun to address the need to integrate digital design in architectural design education, investigating various forms of pedagogical agenda. Design computation and digital design has an influence on the development of theoretical computational and cognitive approaches by various researchers as a foundation for design education and pedagogy (Oxman, 2007).

The researchers and academians all over the world have started to look into this matter with concern, as it has multifaceted outcomes. Contemporary software tools for form generation are partially being used to aid architectural design processes within the early concept and development phases in a studio (Rogers, 2018). However, these are limited to one or two techniques throughout the duration of architectural design projects. Enhancing this simple process with evolving techniques in a generative process and implementing interconnectivity will stimulate a new excitement and era (Arida, 2004), introducing innovative opportunities within all aspects of the architectural realm.

This first wave of practice-driven educational models has brought professional education in architecture to a new frontier. This condition now demands a process of redefinition of the intellectual and cultural frameworks of architecture, as well as for the theoretical foundations for architectural and design education. The need to accommodate the scale of change in professional practice with its new demands of requisite knowledge and skills, presents the design pioneers with a challenge to create theory of architectural education and design pedagogy that acknowledges the scale and qualities of theoretical, professional and technological changes. In the case of such a broad cultural shift, there is a need, first of all, to reconsider the theoretical basis, its related knowledge and its design methods in relation to emergent digital technologies (Oxman, 2007). The foundations of this research are also based on the relationship and connection between architecture and nature. The way the design process is approached is that designers look at nature, specifically organisms or ecosystems, to solve a particular human need; and by doing so, these types of behavioral processes are converted into man-made design solutions. This is imagined as a combination of biology, nature and architecture into one composition (Yazici, 2015).

The biomimicry debate has brought fourth many questions, criticisms and solutions. It almost seems unethical to use architecture to separate man from nature. Today there is a new form of design, has been introduced some time ago which requires a modern man to look to nature for solutions for to architectural and sustainable problems that exist today. These solutions have been around for decades but only recently have their true potential begun to emerge. The question is; can the philosophy behind living organisms be taken and used to aid the development of mankind? Is the process of applying biological principles to understand structures and functionality of biological entities and its connection to man-made design logical. It is "an engineering discipline that emulates nature's design and processes to create a healthier, more sustainable planet" (Amer, 2018: 16 ). This studio work is based on a methodology that imitates nature and, as we all know that nature is a complex cradle of knowledge, so digital tools have been used to pursue the process to bring in the innovation in architecture's form development process.

\section{Teaching Methodology}

The studio consisted of forth year undergraduate architecture students. Their architectural education and the knowledge of design are more or less at a similar level. In terms of technical skills, although some participants attained better skills related to the advanced 3D-Modeling, a majority of them had no experience with digital tools in the past. Introduction to the basic digital tools like Grass Hopper, Rhino and Para Cloud Gem was piloted through lectures in initial classes. 
Rhinoceros is a 3D modeling software that supports its user to precisely model their designs. It is known to be surface modeler for a free-form NURBS. Rhino today is being used for all types of architectural rendering and analysis. Grasshopper is a special plugin integrated with Rhinoceros modeling tool. It is a visual graphical algorithm editor. It is not a programming or scripting tool but still allows architects to visually program their design. While Para Cloud Gem is a tool which deals with different meshes to produce a generative $3 \mathrm{D}$ design. It populates all kinds of geometrical modules or component over different meshes, resulting in the production of complex $3 \mathrm{D}$ and parametric models.

There was a total of thirty students in the studio. Three different projects were introduced to the students. Students were divided into three groups, each group comprising of ten students. The basic aim was to bring variation and variety in the projects. Three projects which are discussed here were to design a vertical scape in Lahore. The major reasons for selecting these three projects for this research study were:

- $\quad$ These projects were completed with maximum details and illustrated step by step research and design process, will in the majority of other projects process was missing.

- To critically analyze and compare the process of design, projects from the same group were selected.

- All these depicted are depicting the abstract transformation of biological research into architecture which illustrated entreat thinking in architecture.

- Lastly, extensive use of digital software and the plugins could be clearly seen in these three cases, as compared to other projects.

The major issue with the methodology used was that the students started using newly learned population techniques randomly on the facades without linking it to the research. Majority of them were so excited to use these newly learned digital techniques in their project that they started to ignore the fact that they have to connect it to the research. The other major problem was the lack of background knowledge of the subject. This method of designing was introduced to the students for the first time during their four years in the Department. There was a varied type of anxiety, fear, and lack of self- confidence that instructors had to witness. Majority of them were confused about both the research- based design process as well as the implementation of the newly learned digital techniques. Introducing this method in fourth-year design studios was intentional: instructors thought that students at this level would not be rigid and will captivate the misunderstandings and apprehensions associated with the use of technologies to design. But it turned out that this approach's rigor, depth, and extensiveness lead to quite a bit of misperception in the student's mind that could be witnessed in their projects. To resolve this major issue it was decided to introduce the theory on the subject in the early stages of undergraduate studies from next year onwards which will hopefully bring a major improvement to the overall learning experience.

Although this approach blatantly put emphasis on process over final results, it was not very well attuned with the students and they kept on focusing on the materialization of architecture, which was purposely not discouraged by the instructors.

It was also felt that investigation of new design vocabularies may require different stages of exploration. This synchronization could be achieved only by liberating the student from prospects that they had learned from the conventional design approach, which was not fully possible to achieve during this short four-month semester.

Students were provided with the following four sets of instructions to work on as part of the course:

1. For the first three weeks, the students were told to explore and research on the natural processes or phenomena of their interest. They were supposed to study in detail each aspect of the various phenomena in detail.

2. Then they were instructed to design an architectural module by narrowing down the research and were taught to mind map their research.

3. Next the student were asked to enlist and specify site research and identify environmental constraints.

4. To further develop their design and incorporate the enlisted constraints in their architectural design, they were then directed into using their research and find solutions to the defined problems.

To help them do the above, it was made obligatory to use digital tools including Rhino, Grasshopper and Para Cloud Gem. Majority of the students preferred Para Cloud over 


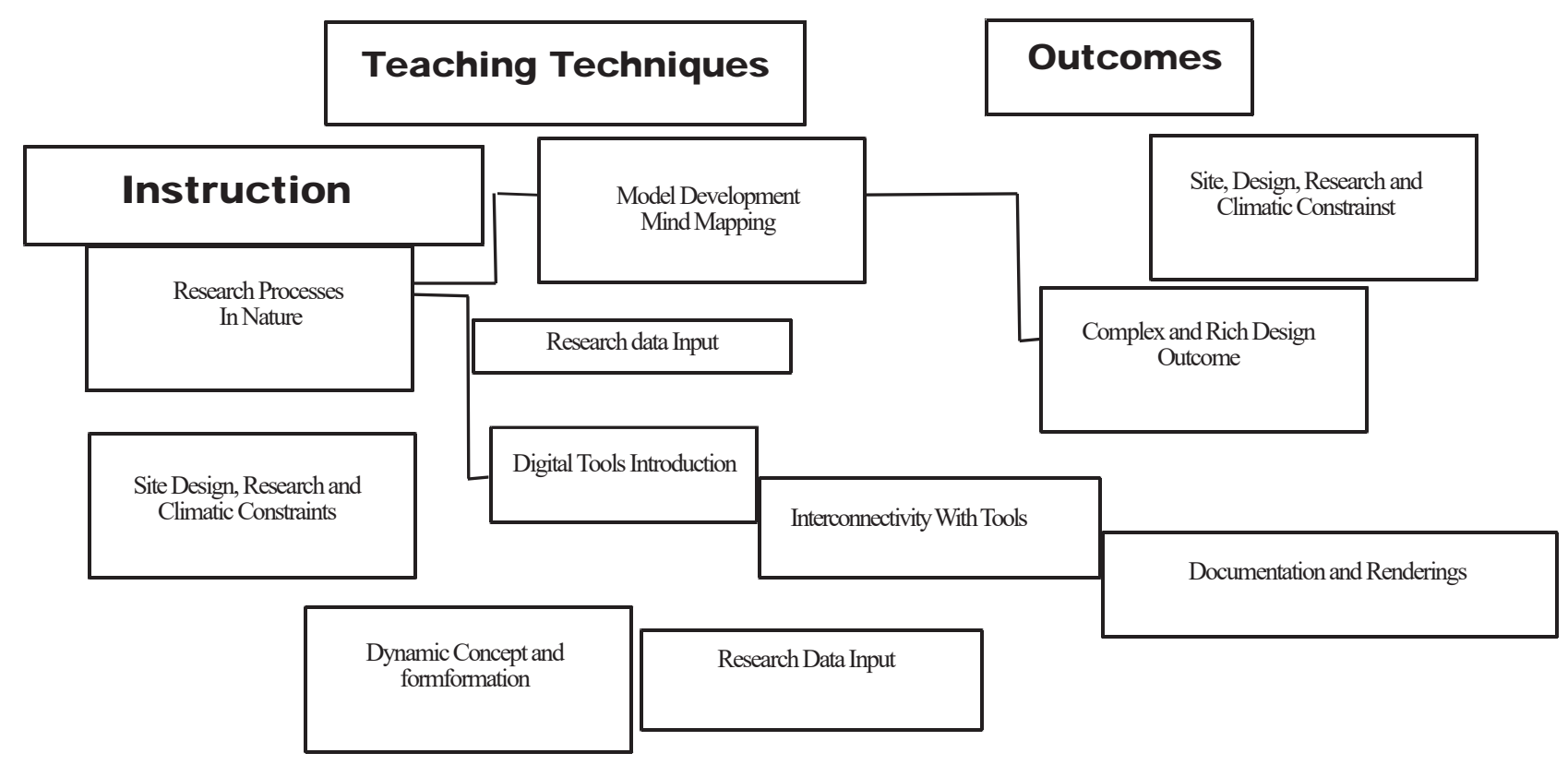

Fiure 1: Proposed framework for the design studio.

Source: Author

Grasshopper, but few did a great job exploring it. Figure 1 shows the framework for this studio with inputs as instructions, teaching methodology and the outputs as the final project. The proposed methodology diagram treated the tools as a central component of interaction, as the design cannot be complete without them. This framework focused on implementing innovative procedures singularly and collaboratively to invoke new experiences and definitions of architectural designs.

\section{Limitations and Challenges}

Design studios are a vital learning experience for students. The aim of this particular design studio was to explore the concept of Bio-Mimicry to produce varied innovative results and to analyze various techniques that the students adopted to reach a final product. This studio's working methodology additionally included enhanced courses and seminars, based on digital tools handling, which was fed into the studio. This studio experimentation went beyond teaching pure skills and necessitated reflection upon the creation of knowledge, which was analyzed and studied by the instructor to develop a framework for digital architectural pedagogy. However, a gap was witnessed between skills training and the application of knowledge within this studio environment.

During the final presentation some of the students were not able to identify how they arrived at their solution and there was visual imprecision in the design connectivity.
Another concern was to make sure that students transformed the research knowledge that they had gained and apply it accurately to the final product, as most of them were not keen in using the knowledge and skills they had gained earlier. Majority of the students had a tendency to change their learning foci to the previous semesters' learning. This issue was addressed by linking the design decisions with research attributes and inquiring about how to take informed design decisions. The assimilation of research knowledge allowed participants to understand the impact of each decision taken on the design of their project. This helped in clear documentation of the design process and research findings. The participants also developed and communicated their understanding of architectural design by utilizing their skills within the design-studio environment, which was aided by regular discussions with the instructor and enabled the students to think about design solutions in alternate ways.

Although the studio instructions were the same for every student, yet, the outcomes were varied, different and unique. Two major approaches were distinct, where one approach considered the relationship between nature and architecture, and was based on the inspiration of architectural form through natural forms and imitation of its properties, such as patterns. The other approach was based on learning's from natural forms, by trying to understand the systems and processes in the environment. 


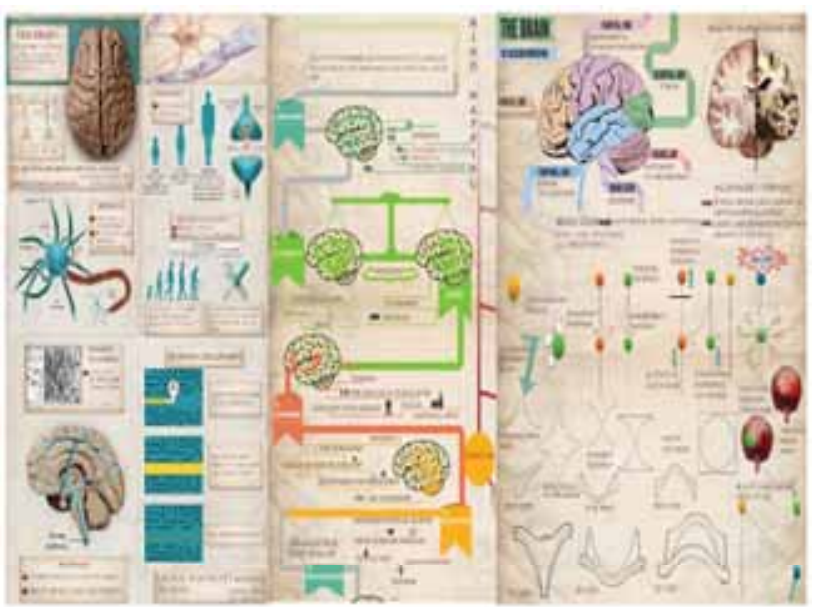

F igure 2: Presentation panel site constraints.

The students were instructed to propose design of vertical landscape in the city of Lahore. The processes which the students investigated varied from neuro-plasticity to the structure of glass sponge, to process of pollination. Students with different technical skills used different mediums to present their ideas, by specifying constraints, rules and relationships of the system with architectural needs on site. The result achieved outputs were grouped into two different categories; a) generation of morphological and organizational relationships and b) mimicking of properties and processes in nature.

\section{Exploration 1: Neuro-Plasticity}

In the chosen project the student researched the interesting process of neuroplasticity and attempted to design a vertical landscape tower inspired by the process of neuron morphology. Neuroplasticity can be defined as the brain's ability to change, remodel and reorganize for the purpose of attaining better ability to adapt to new situations. Neural networks are not fixed and they occur and disappear dynamically throughout life, depending on experiences. While one repeatedly practices one activity such as a sequence of movements or a mathematical problem, neuronal circuits are being formed, leading to better able to perform the practiced task, with less waste of energy. Once one stops practicing a certain activity, the brain will redirect these neuronal circuits by a much known 'use it or lose it' principle (Demarin, 2014).

The phenomenon of architectural interpretations of nature was studied and then its architectural interpretation was evolved by applying it to real site constraints. The initial research was narrowed down to designing an architectural module based on the basic principles of neuroplasticity. The

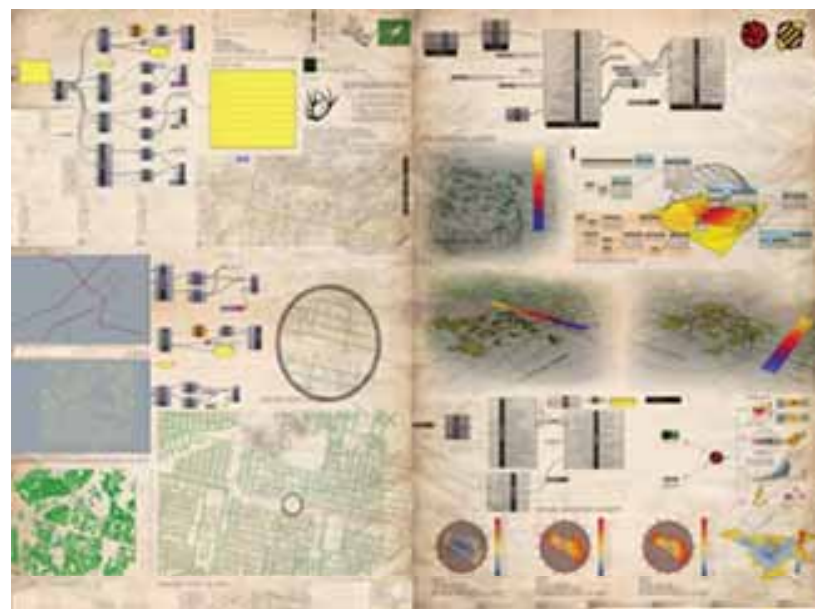

F igure 3: Detailed environmental analysis presentation.

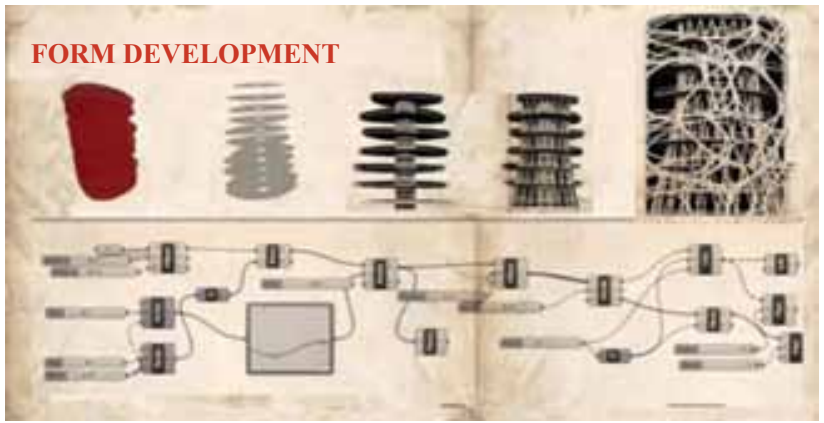

F igure 4: Development process presented using Grasshopper function.

environmental, structural and social constraints on the site were also analyzed (Figure 2). This particular student worked on the lady bug and honey bee models ( Grasshopper Plugins for Environment Analysis) as inspiration using Grasshopper Plugins for Environmental Analysis. The student performed the detail environmental analysis (Figures 3). The selected site was located at Phase V Defense Housing Authority Lahore's commercial area, as the weather of Lahore is hot and humid for almost eight months, the façade was designed in a way that it reduces solar gain. The double skin façade was inspired by neurons connectivity and linkages and architecture solutions were provided to avoid direct sun and to provide a dynamic play of shadows. As the site was located in one of the posh residential communities of Lahore the provision for privacy for residents was important and a buffer space was provided in the design. The project resulted in vertical tower design, optimal size structural columns and reduced floor size. The structure was specifically designed to handle the wind pressure at the top for structural stability (Figures 4). The floors were designed with the computer programme called Grasshopper so that the model could be rotated and reduced according to the needs and constraints of the project. 


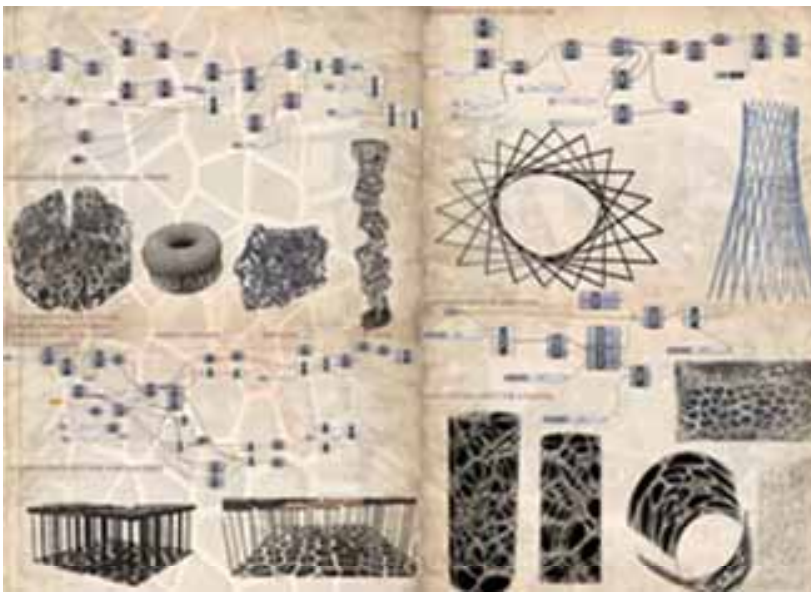

Figure 5: Structure and Facade development process using Voronoi Technique in Rhino

The main concepts of this process investigated were neural changes and morphosis, which could be applied on design variables, and which could be altered according to the studied constraints, programmatic requirements and structural needs, to satisfy the design criteria.

The method adopted resolved around the problem of distributing a given amount of material in a design domain, subject to load and support conditions, by maximizing the stiffness of the structure. It calculated stresses affecting the geometry, based on specific boundary conditions and loads, and organize efficient structural arrangements. The students also investigated neuronal migration, which is a process in which neurons travel from their 'place of birth' in fetal ventricles, towards their final position in the cortex and the process was used for the development of the skin, with the use of Voronoi (Voronoi diagram is a partitioning of a plane into regions based on distance to point in a specific subset of the plan) (Figure 5). The tower form was then shaped according to the forces of pressure and forces existing on the site.

In the process, CAD and visual programming tools like Grasshopper, KARAMBA, (Karambais is an interactive, parametric finite element program which lets one analyze the response of 3-dimensional beam and shell structures under arbitrary loads) Elk, (Elk is a set of tools to generate a map and topographical surfaces using open source data from Open Street Map.org) USGS Heron, (Heron is an addon which enables the import of GIS data from various sources into the Rhino/Grasshopper environment, located, scaled and cropped based on an Earth Anchor Point and a clipping polygon) and Lady Bug were used for environmental analysis. The boundary conditions, including loads and support regions

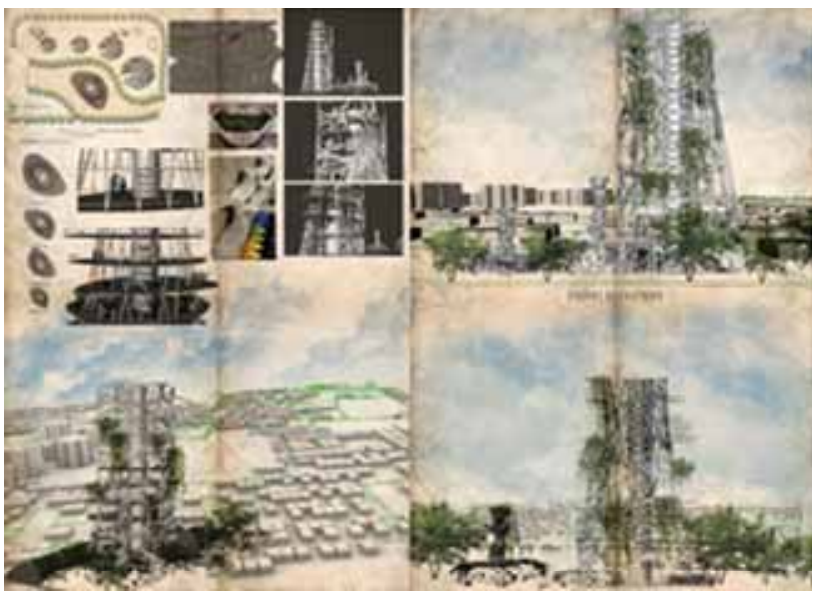

Figure 6: Final Visualizations of the project.

of the tower, were defined. During the process, a series of iterations were also developed.

Grasshopper -a plugin for Rhinoceros software- was used in the process as a parametric modeling tool to develop drawings of student concepts. The plugin is mainly an algorithmic modeling tool for Rhinoceros which provides a visual scripting interface, allowing users to build their three dimensional models without prior knowledge of complex programming languages or scripting experience. It is worth mentioning that Grasshopper was used as a tool in the design process; it can be an extension of the process, which enables architects to create innovative new possibilities and to test a variety of new ideas in a quick and easy way. The final visualizations of the project can be seen in Figure 6.

\section{Exploration 2: Glass Sponge}

The second significant exploration was based on the concept of a glass sponge. The glass sponge is a deep water animal identified for its convoluted glass-like skeletal structure. This student studied the structure of a glass sponge, its morphology and movement and then interpreted the architectural design for a vertical landscape in the city. Sponges are interesting deep sea creatures, their unique characteristic begins with their mineral skeleton that is composed of silica (glass) spicules and continues with their extraordinary capacity to create astonishing glass structures. The morphology of these creatures is also incredibly different from other species due to the structure of their cells and the three-dimensional symmetry (Barbanera, 2017). The architectural module was derived by narrowing down the research on sponges with the help of mind mapping shown 


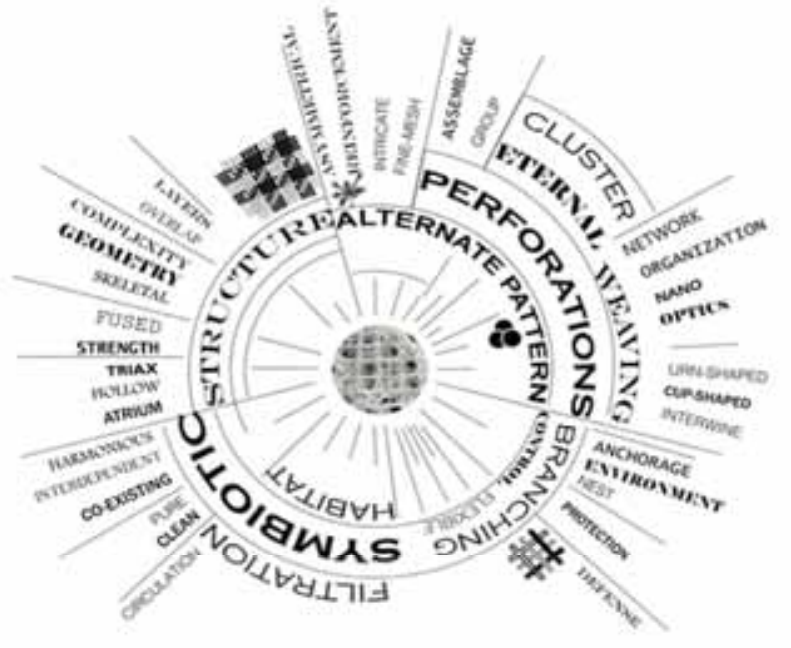

Figure 7: Mind Mapping to develop the module.

in Figure 6. Glass sponges spicules interwined together to form a very fine mesh which gives it's body a firmness which is usually not found in other sponge species, and allows glass sponge to survive at greater depths in the water column. Learning from the sponge's structure which is a thin-walled, cylindrical, vase-shaped tube with a large central atrium, architecturally, this detail was adapted to design the module (Figure 7). A detailed study and site survey was conducted by the student for user analysis, traffic, and climatic analysis to chalk down the constraints (Figure 8).

The body structure of glass sponges is a thin-walled and tubular form with a large central atrium. These creatures reach larger heights, turning and twisting and forming bends. The base of the sponge is usually narrower than the top. It is also seen that the glass sponge has a diagrid structure (Chu, 2010). To interpret this in design, full-length columns were designed at the periphery of the tower with cross diagrid enclosing the structure. Structure of the fiber in the glass sponge shows layers that give it rigidity and prevent it from buckling. This method was applied to the building structure by using various layers to ensure stability in design. Column bracing was proposed in the design which had alternate reinforcement, and used less material but offered the same amount of strength as that of a fully braced column. The shape of the architectural and structural form used the Voronoi Diagram, which is one of the most important trends in seeking new forms of expression in architectural design. In shaping contemporary architecture, the Voronoi Diagram is a synergetic solution for a new generation in architecture (Nowak, 2015). This tessellation was used to design the architectural façade of this tower (Figure 9).

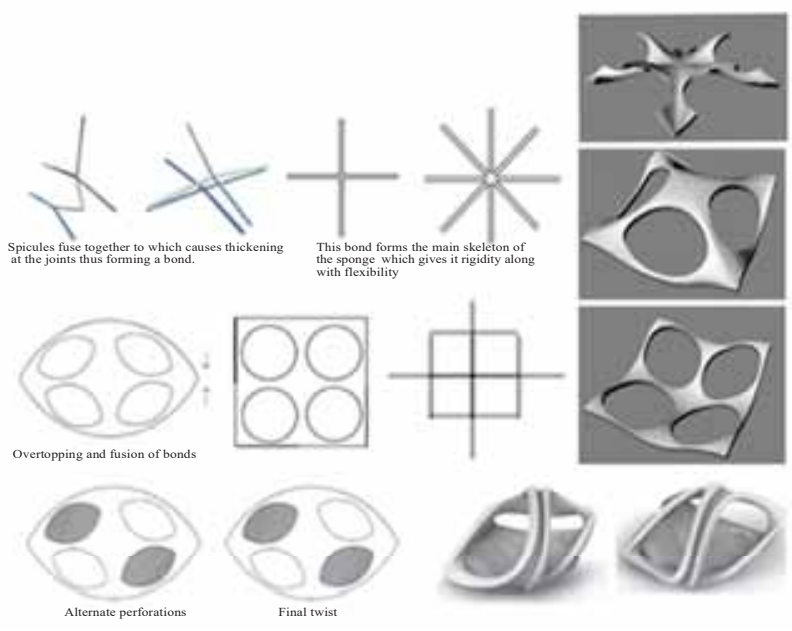

F igure 8: Development of the module.

The use of Voronoi diagrams was visible in the design. Open spaces, gardens, theaters, and other similar space were placed in the individual cells of the Voronoi diagram. These diagrams were used in searching the immediate neighborhood, determining the function of the position (vertices), finding the largest empty circle (cell), and path planning (edges). These diagrams were also used in determining the optimal deployment of infrastructure or functional separation (Nowak, 2015).

Here the Voronoi technique was used to design the double mesh skin that helped in filtering direct sunlight. When viewed from the top of the glass sponge it was composed of the Voronoi pattern. This pattern contained many nodal points which enhanced the connectivity, hence it was used to design the master plan and pathways. The glass sponges are usually found clustered in nature. They cluster around in such a manner that they usually have a common center point. Inspired by this phenomena the three towers were placed in a way that they depicted clusters and had a common attraction in the center (Figure 10). The module was also designed in a pod-like form with a double envelope to control the direct sun. Their cells were fused together, resulting in one large multi-nucleated cell that is folded around a mineral skeleton.

Glass sponges can use this fusion to spread electrical impulses to regulate their filtering actions. The triatomic symmetry is made of three axes that are perpendicular to one another, creating a three dimensional structure (Chu, 2010). The same principle was adopted here to design the structural core and façade of this landscape tower. 

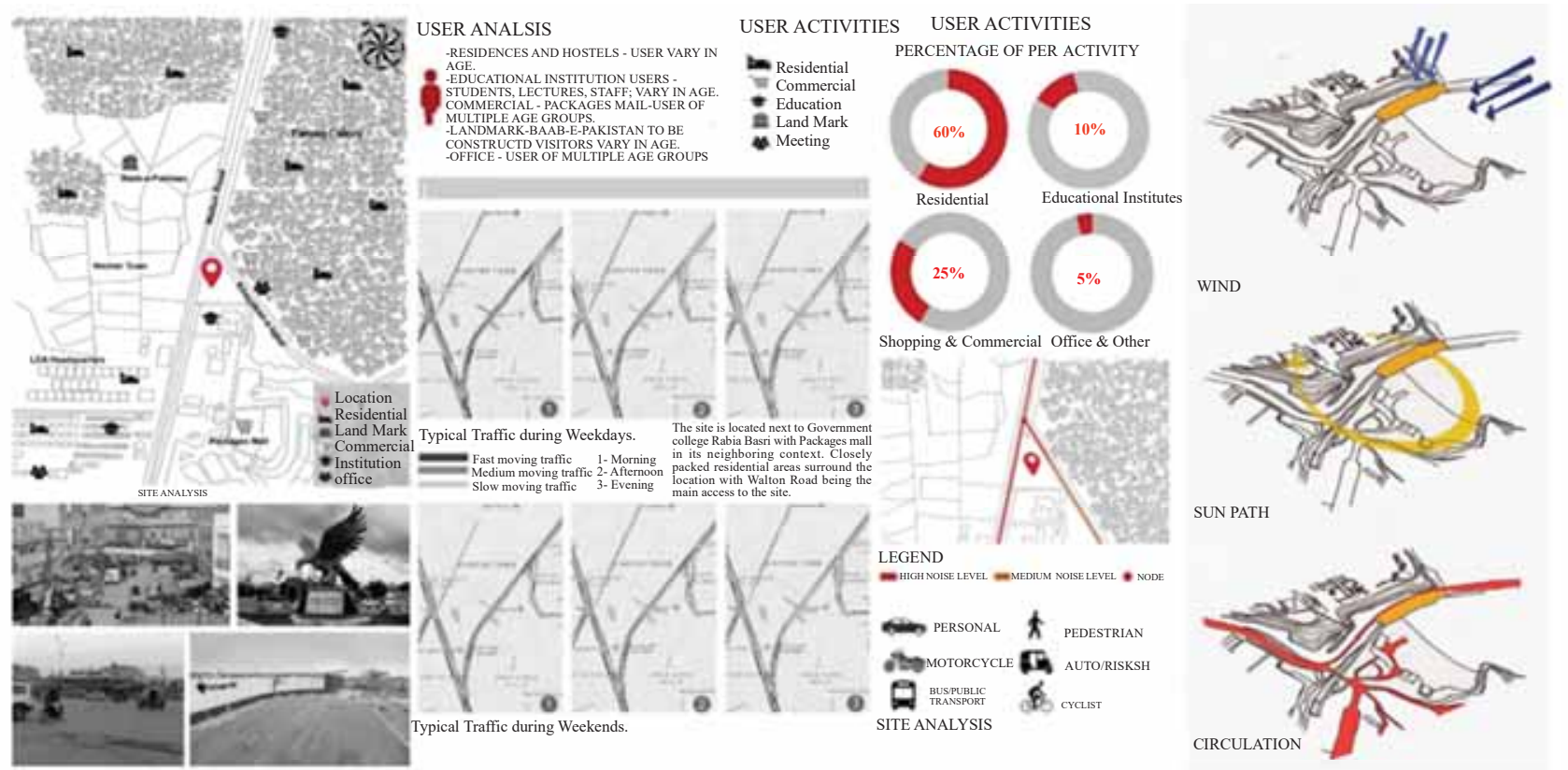

Figure 9: Details of site analysis to study various constraints.
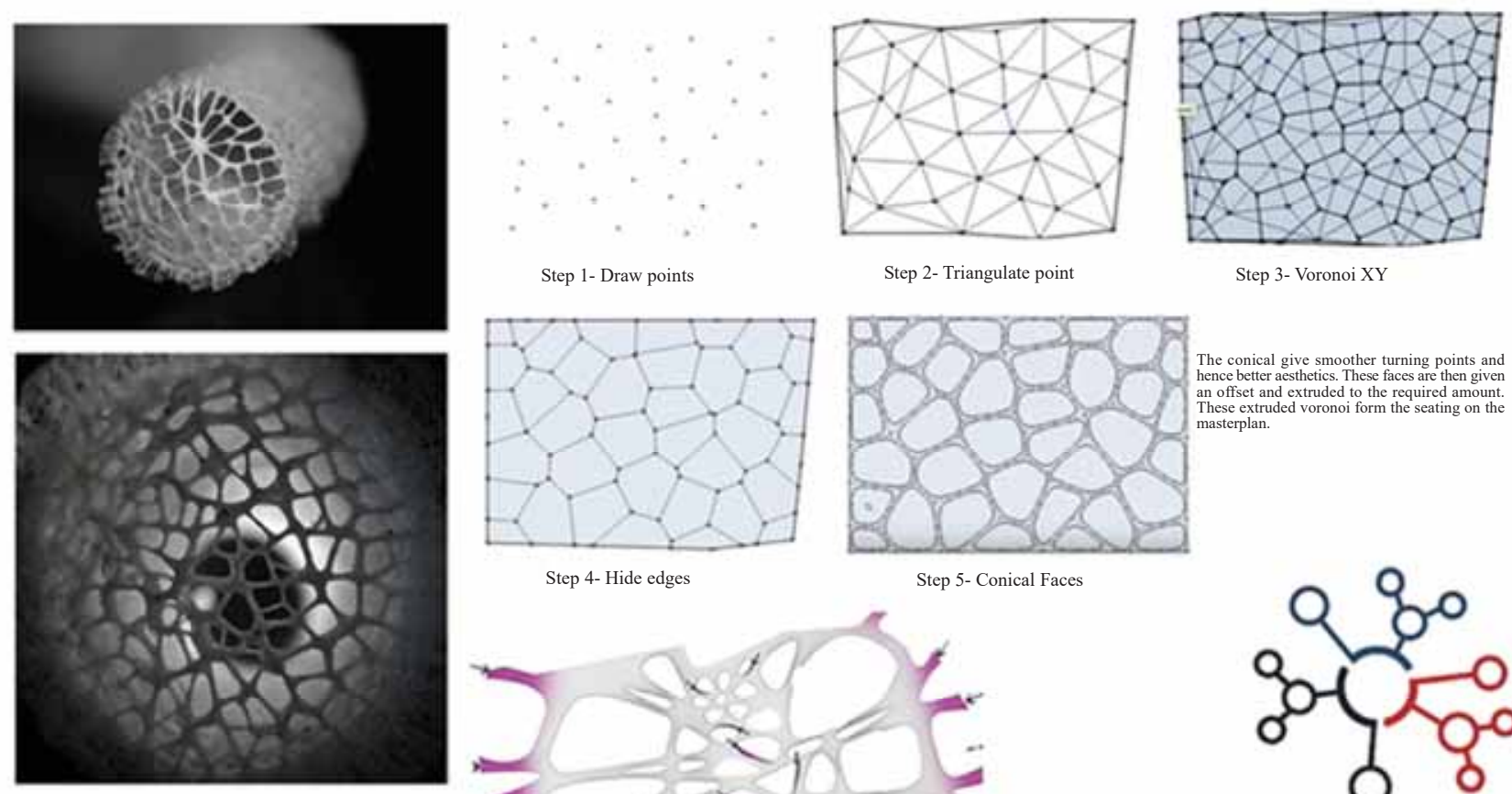

The top of the glass sponge is composed of the voronoi pattern.
This pattern contains a great deal of nodal points which thus makes the possibility of interaction greater than in any other pathway
design. Thus this pattern is proposed on the footprint of the site excluding the footprints of the building.

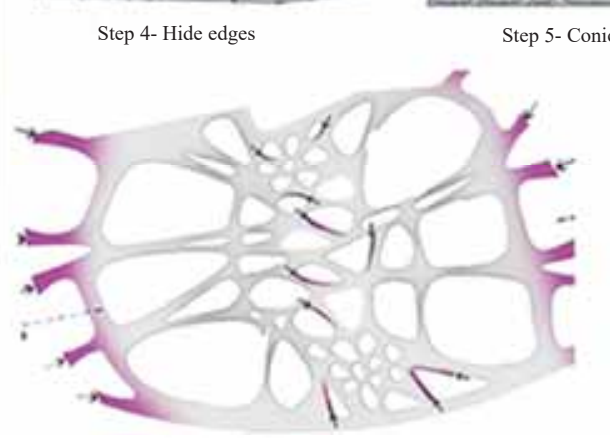

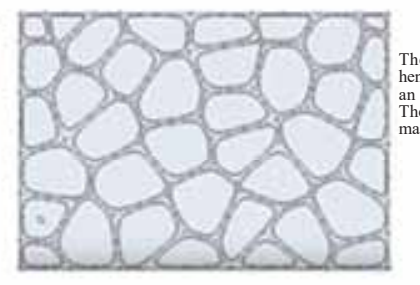

Step 3- Voronoi XY

The conical give smoother turning points and hence better aesthetics. These faces are then given an offset and extruded to the required amount.
These extruded voronoi form the seating on the
masterplan.

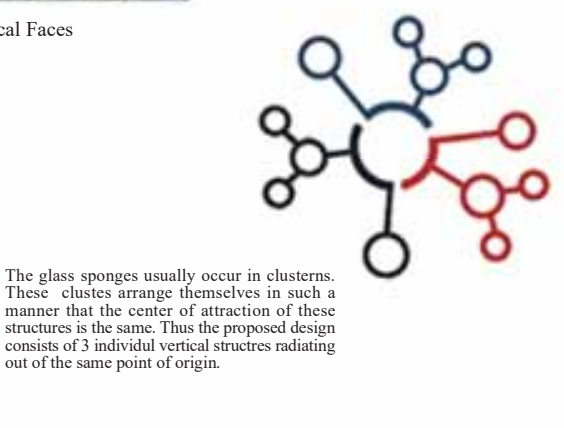

F igure 10: Details of using Voronoi Technique to develop the façade of the tower as a top of a glass sponge, composed of Voronoi pattern in nature. 

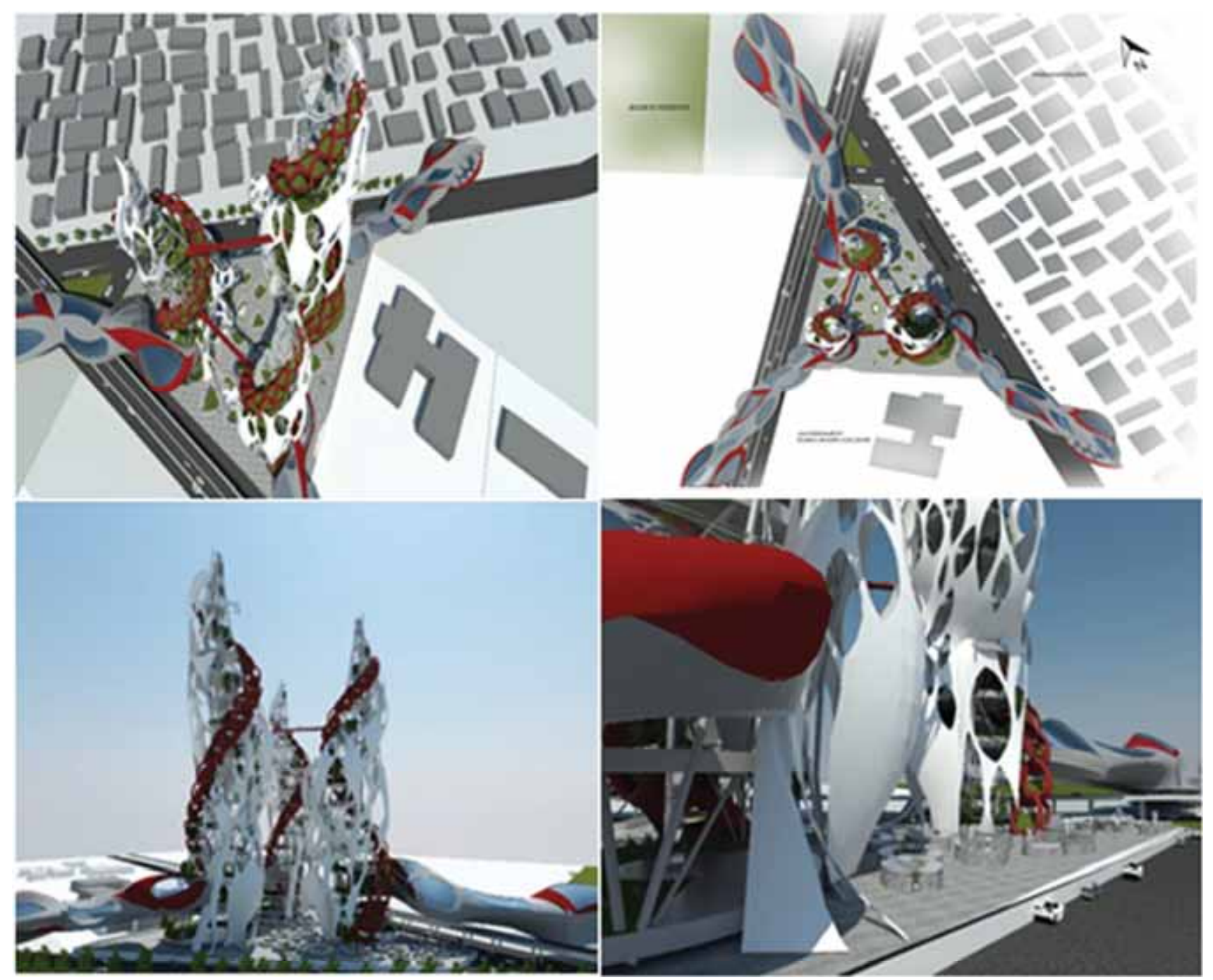

F igure 11: Architectural Visualizations of the landscape towers inspired by the Glass Sponge Morphology.
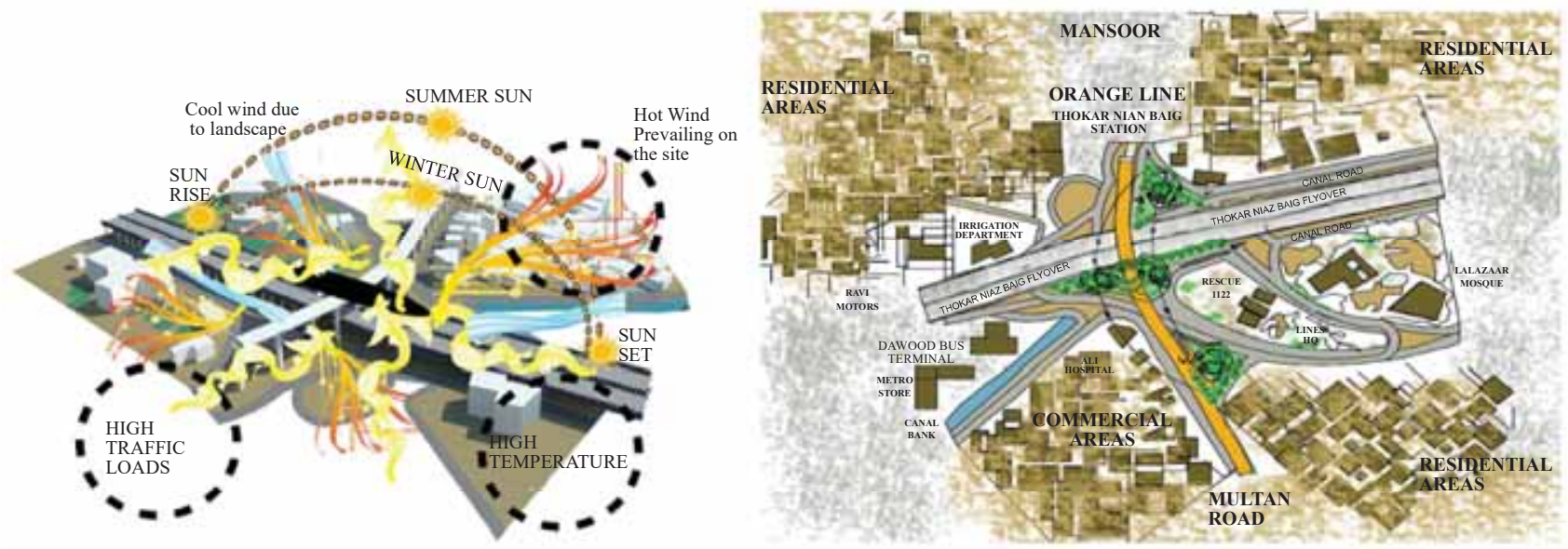

F igure 12: Climatic analysis for site constraints. 


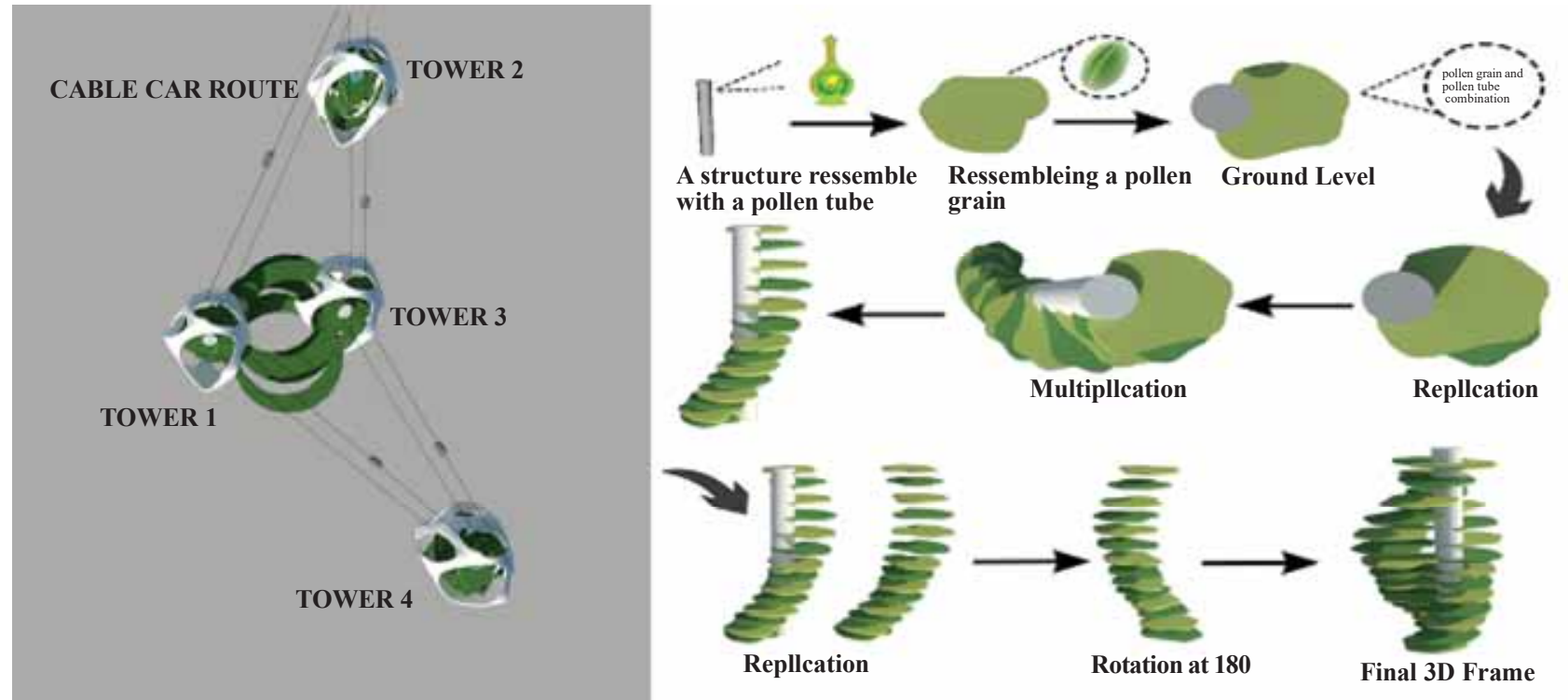

Figure 13: Step by step process of Evolution of the form for the landscape tower.

\section{Exploration 3: Pollination}

The research for the next project was conducted on the process of pollination. After exploring different phenomena linked with pollination and studying its types, a mind map was drawn to design an architectural module. The design challenge was to solve the problems associated with urbanization, such as pollution and global warming, by designing a vertical landscape. The student selected the site of Thokar Niaz Baig in Lahore to design vertical landscapes. This site was a highly urbanized area, had a high level of noise pollution and heavy traffic loads, and badly needed some green breathing space. Different issues were identified regarding the site, which were related to climate and social life (Figures 11 and 12).

The process of pollination involves a pollen grain being transported to the stigma, where it germinates and its pollen tube moves down the style to the ovary. The gametes are held within the carpel and later produce the embryo. Different types of animals are responsible for this pollen transfer and act as part of the pollination process. Some of these include bats, birds and even land mammals, but the most common pollinators are insects (Dar et.al., 2017).

The captured pollen initiate a complex series of events that can lead to the hydration and germination of the pollen grains, and ultimately to a dispersion of the pollen tube and fertilization. The master plan of this project was inspired by the process of hydration of pollen grains (Figure 12). According to the site constraints, there was too much noise,

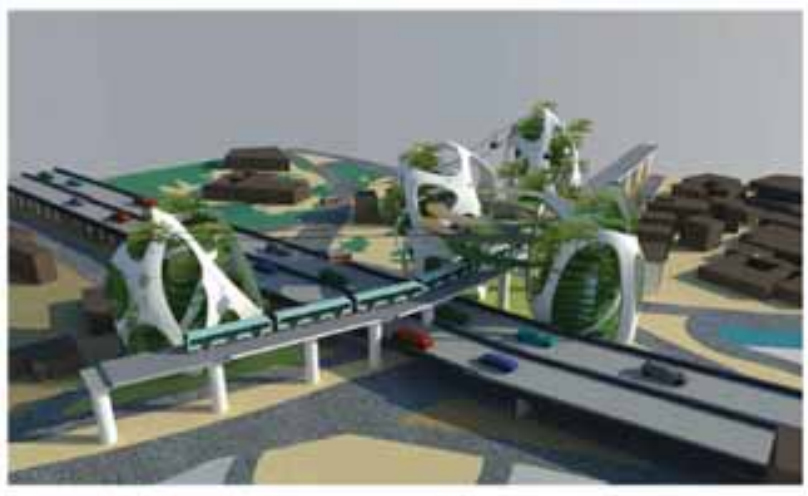

r Igure 14: Architectural visualization of the three vertical landscape towers on the site.

pollution, traffic load, dust and urbanization on the site so one structure couldn't address all the issues. Thus, four vertical landscape towers were designed on the site in the near vicinity. The hydration cycle of pollen grains helped the student to vary the sizes of the four towers designed on $2 \mathrm{n}$ formula, because of the availability of unequal land pieces. Inspired by this cycle, size variations in these structures were calculated that resulted in interesting forms of the towers (Figure 13). An architectural module was designed, based on the concept of morphological characteristics of pollen grains, which were categorized into different groups (Figure 14). The three dimensional form accommodated the different attributes of the tower taken from the process of pollination and the process was interpreted by using terms like repetition, reproduction, copy, deflection, alteration, shift and replication. Exposure to the outer environment and feeling of grandeur and 


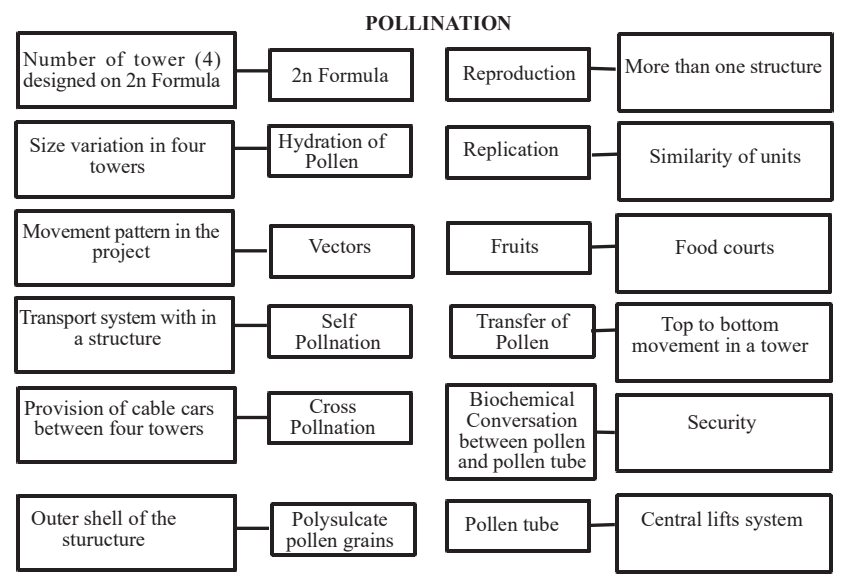

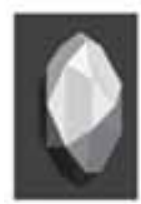

Hedra

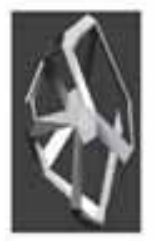

Squeeze

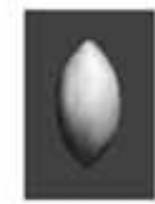

Turbo Smooth

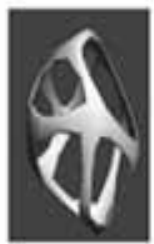

Turbo Smooth

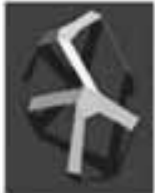

Editable Poly

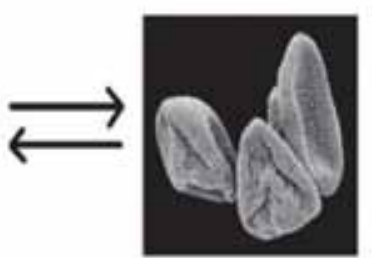

Poly Sulcate Pollen grain

Figure 15: : Relationship diagram showing research and evolution of the module inspired by PolySulcate Pollen grains

openness at the same time provided the feeling of security, surveillance and sense of territory in the design. Huge perforations in the outer skin of the building allowed proper sunlight to come inside for enabling the plants to grow green. The master plan mimicked the process of pollination. These four towers were linked together through bridges and cable cars for easy transportation. This student limited herself to the use of 3D Studio Max and Paracloud Gem softwares.

\section{Discussion and Analysis}

This studio was initiated with the objective to explore organisms and nature with a particular characteristic and processes, which the students found intriguing through a process of discovery. When the students were given freedom to choose and select the domain of their study area, they automatically took the responsibility for maintaining the veracity of nature's designs. When the students began to understand the selected organism and then process, it made their process of abstraction more trustworthy. Use of abstraction and mind mapping and an open-ended process of interpretation, helped students in translating the biological characteristics into a unique design approach. The strength of this studio's approach was based upon this process of abstraction and perception with the help of nature's lens. The students were guided to emulate nature not only from different perspectives, but also at different scales. The focus was on the process of discovery and design rooted in fundamental respect and understanding of nature's design.

The objective of the studio was to define a research-based design framework, hybridizing both the digital and the biological approach to design. The idea was to discover a higher consciousness of digital tools and indulge the possibilities of this fusion to obtain creative methodology for architectural design. This was attained through a series of steps and the process was recorded with the help of architectural diagrams.

The teaching started by introducing different components of the digital theory. Students were introduced to all three tools with detail visual demonstration. Related literature on digital architecture and biomimicry was provided for better understanding of the subject.

Researching and selecting about natural processes and organisms leads to the necessity for having a critical approach towards abstraction for design, in the sense that in-depth knowledge can be acquired to create a link for producing a module first and then building on it later on. The drawing of architectural drawings was emphasized for a clear understanding of whether the solution proposed provides solution for the constraints in an optimum way, or could there be other alternatives. To produce this, the students were given small exercises whit a critical approach, ranging from mind mapping and morphological diagrams of the module. The students were also asked to produce module variation diagrams on different meshes and with different tools to select the best ones for their final product. Nature inspired thinking has been very fascinating as it can be witnessed by the creativity and variety present in student's projects. Both students and instructors found this way of thinking attuned with the nature of architecture design process. It was an exclusive experience to work with students throughout the studio using this methodology to design as it helped in developing their analytical thinking which at the same time appraised the use of digital design tools. 
When students had developed a fully detailed virtual model it provided them the freedom to experiment with a variety of options. With the help of this exercise it was also established that these tools can reduce the time to explore the variation in design. Students also learned to create and handle the relations between different components of design because of the availability of many options. These variations were not only present during the form-finding stage, but also at the research level. These digital tools and techniques opened up new possibilities for the student. Thus, there is an urgent need to explore studios like these to propose a new framework for architectural pedagogy. Studios structured in this way can reduce the gap between digital architecture education and its practice.

These projects also taught students about how to handle the real constraints, challenges and potentials of site digitally. One advantage of the approach introduced here was that it did not rely on specific knowledge and skills to be able to produce the design; using this method also provided myriad solutions for the same problem. The pedagogy adopted help raise a number of question.

- Does the field of architecture need a prominent engagement with research and experimentation in this digital era?

- How does one define the role of Computer Aided Architectural Design (CAAD) and its link to architectural pedagogy?

- How can research findings from architectural studios find their way to practice?

One of the primary outcomes of this experimentation was the creation of awareness of the very important fact that technological advancement in architectural studios are getting overpowering with time. Innumerable members of the public outside of an architectural education remain somewhat unaware of the various technological advancements available, which can be implemented collaboratively within an architectural design methodology. Hence, the new framework for design education should make these tools an important component. Learning outcomes of this studio also included an introduction to the philosophy of biomimicry to architecture, introduction to new digital tools and a methodology to conduct research with the help of mind mapping and translating it into architecture that resolves real-life problems. It must however be highlighted that digital tools were not explored to their fullest and ideas could have been developed further.
This method of designing was introduced to the students for the first time during their four years in the department. There was a varied type of anxiety, fear, and lack of selfconfidence that instructors had to witness. Majority of them were confused about both the research-based design process as well as the implementation of the newly learned digital techniques. Contemporary software tools for form generation were partially used to aid architectural design processes within the early concept and development phases in the studio. However, these were limited to one or two techniques throughout the duration of architectural design projects. Enhancing this simple process with evolving techniques, in a generative process, implementing interconnectivity can stimulate a new exciting era, introducing innovative opportunities within all aspects of the architectural realm (Rogers, 2018). On the other hand, the importance of learning from nature should also be given importance in any recent design pedagogy as natural processes are very complex and a great reservoir of knowledge. When students studied a process in nature, they were intrigued by the amount of information they gathered about connectivity, interaction of formations with in a system.

The major issue with the methodology used was that the students started using newly learned population techniques randomly on the facades without linking it to the research. Majority of them were so excited to use these newly learned digital techniques in their project that they started to ignore the fact that they have to connect it to the research.

The other major problem was the lack of background knowledge of the subject. Introducing this method in fourthyear design studios was intentional as the instructors thought that students at this level would not be rigid and can captivate the misunderstandings and apprehensions associated with the use of technologies to design. But it turned out that this approach's rigor, depth and extensiveness lead to some misperceptions in the student's mind's which was witnessed in their projects. To resolve this major issue it was decided to introduce the theory on the subject in the early stages of undergraduate studies from next year onwards, which will hopefully bring a major improvement to the overall learning experience.

Also, investigation of new design vocabularies may require different stages of exploration. This synchronization can be achieved only by liberating the student from prospects that they have learned from the conventional design approach, which was not fully possible to achieve during this short four-month semester. 


\section{Conclusions:}

The projects produced as a result of this studio were very creative and unique in their own way. This exercise in the studio asserted that biomorphic principles should be considered in the design process and incorporated in architectural design education, along with the help of modern design tools. Based on the results, it can be concluded that students learn from natural systems in three different ways which include, generation of morphological and organizational relationships, translation of processes in nature followed by the process of adapting to the design. The results also showed that this technique and methodology had abiding effect on developing skills. Student developed their selfreflection, critical and creative thinking and problem-solving techniques. The recommendation included the need to consider this topic as a basis of the design education system. The major struggle encountered in the process was embracing Bio-mimicry and use of digital tools together, which was a novel concept for the students and they had some level of difficulty in working with it.

For the upcoming studios, the intent will be to teach this method in a more elaborate and challenging way, by giving students real site projects and encouraging them to work with multidisciplinary groups of people and to keep the focus on research interpretations. It was noticed that little or no research is present that examines or validates the claim that framing of design development using digital methods enhances this process. This studio, therefore, attempted to combine digital design methodologies within the conception of architectural design, to analyze and reframe methods of design thinking. The inspiration with nature is an important aspect in the design of architecture. It is particularly important when designing bionic structures based on the formation of biological structures. The contemporary line of thought is to understand the processes occurring in nature and describe them appropriately in order to apply these models in technology, including architecture. The development of digital tools currently used in architecture allow for the portability of designs found in nature to the spatial and structural surface shaping. New methods change the way people work in architectural design. This exercise provided students with a deeper understanding of the processes in nature and to come up with solutions that are unique.
In order to build up a theoretical discourse around digital and natural processes and their relationships, the partakers used digital tools that allowed them to create and express their designs. With these tools, users could develop the expertise to engage creatively in design. Digital applications allowed the study of architectural conditions in a threedimensional environment, rather than the commonly used two-dimensional or layering techniques, which made the studio more intriguing and interesting, as the underlying concept of digital modeling was based on data, variables and their relationship to other entities, which then produced variations in design.

This research paper's methodology allowed the students to work side by side with digital tools to interpret the research they had conducted. Within the framework the student could always revert back to a singular point within the system and change the design direction as desired, as the interconnectivity and dynamic style of the methodology allowed this flexibility. The dynamic implementation of the digital tools created the desired complexity and richness of the design.

\section{Acknowledgments:}

I am highly obliged to my students, Ghana Ahmed, Kanza Khalid, and Amara Fatima for working hard on the projects, to the Department of Architecture, University of Engineering and Technology for providing the resources and the Studio Arch-Architectural Consultancy Firm which was used for research. 


\section{REFERENCES}

Amer, N., 2018, "Biomimetic Approach in Architectural Education: Case study of Biomimicry in Architecture' Course", Ain Shams Engineering J ournal, Vol. 9

Arida, S., 2004, “Contextualizing Generative Design”, S. 1.; Thesis, Massachusetts Institute of Technology, Department of Architecture.

Barbanera, A., 2017, "Extreme Marine:Bangor University student perspectives on extreme habitats", viewed 24 October 2017, from https://extrememarine.org.uk/2017/10/sea-glass-sponges-the-immortal-architectures-of-the-deep-seas/

Chu, J. 2010, Biological Patterns and P rocesses of G lass Sponge Reefs, Newfoundland: Research Gate.

Dar, A. S, G. I. Dar et.al, 2017, "Pollination and Evolution of Plant and Insect Interaction”, J ournal of Pharmacognosy and P hytochemistry, 6(3): 304-311.

Demarin, S. M. 2014, "Neuroplasticity”, P eriodicum Biologorum, 116(No 2), 209-211.

Nowak, A., 2015, “Application of Voronoi diagrams in contemporary architecture and Town Planning”, Civil Engineering, Urban Planning and Architecture Journal of Warsaw University of Technolog, Vol 9.

Oxman, R., 2007, Digital Architecture as a Challenge for D esign Pedagogy: Theory, Knowledge, M odels and M edium, Israel: Elsevier Ltd.

Rogers, M. A. S., 2018, “Digital Culture-An Interconnective Design Methodology Ecosystem”, Caadria, 197.

Yazici, S.,September 2015, “Course on Biomimetic Design Strategies”, eCAADe 33.s. 